\title{
New perspectives for the Silicon-On-Diamond material
}

\author{
S. Lagomarsino, G. Parrini, S. Sciortino*, A. Fossati \\ INFN and University of Florence, Italy \\ M. Citroni ${ }^{a}$, G. Ferrari ${ }^{a}$, F. Gorelli ${ }^{a b}$, M. Santoro $^{a b}$ \\ ${ }^{a}$ LENS, Florence, Italy \\ ${ }^{b}$ CNR-INFM CRS-SOFT, c/o Università di Roma “La Sapienza," Rome, Italy \\ G. Molesini, M. Vannoni \\ INFN and INOA-CNR, Florence, Italy
}

A. Marras ${ }^{c}$, A. Scorzoni ${ }^{c d}$

${ }^{c}$ IMM-CNR, Bologna, Italy

${ }^{d}$ INFN and University of Perugia, Italy

\section{A. Ranieri}

INFN and Politecnico di Bari

L. Berdondini, F. Brandi, R. Carzino, A. Diaspro, M. Scotto, B. Torre

IIT, Genova Italy

\begin{abstract}
A recent laser bonding technique has been developed to prepare silicon-on-diamond material. This method allows the independent choice of the quality of the silicon and diamond plates to be processed. This opens a wide field of applications for silicon-on-diamond devices. We present and discuss the laser technique and the experimental results achieved. Two new projects involving relevant issues both in high energy physics and in biological applications are also presented.
\end{abstract}

9th International Conference on Large Scale Applications and Radiation Hardness of Semiconductor Detectors

30 September - 2 October 2009

Florence, Italy

${ }^{*}$ Speaker. 


\section{Semiconductor On Diamond Research}

Several works have been reported in recent years on the implementation of the widely diffused Silicon On Insulator system[1] with the Silicon On Diamond (SOD) concept[2, 3], due to the extremely favorable thermal properties of diamond. GaN-On-Diamond has also been proposed and commercialized for applications in power electronics[4].

Most of the research on SOD fabrication are based on Chemical Vapor Deposition (CVD) of polycrystalline (pCVD) diamond on silicon[5, 6, 7]. This approach can be satisfactory as far as diamond is used as an electrically insulator and thermal spreading material. However, diamond is intensely investigated also for its radiation hardness, its solar blindness and its nearly tissue equivalence, for applications in high energy physics [8], space experiments[9] and clinical dosimetry[10]. Furthermore, its very favorable biocompatibility and electrochemical properties[11, 12] make it a unique material for interfacing living cells. We envisage SOD applications, both in radiation detection and in biophysics, through the implementation of monolithic, rugged, low-noise systems where diamond is either the active sensing material or the biological interface and silicon is the carrier of the integrated electronics.

To this purpose, it is necessary to select independently the properties of the two materials, a goal which is out of reach of the CVD growth techniques. Direct bonding of independent silicon and diamond samples have been unsuccessful in the past[13, 14]. Only recently a bonding method for silicon-on-diamond preparation has been presented[15], which is based, as in the case of the $\mathrm{GaN}$ on diamond structure[4], on a nanometric buffer layer interposed between the two materials.

We have developed a laser enhanced method capable of a true direct bonding of silicon and diamond by locally melting the materials to create a SiC/amorphous silicon interface of less than 100 $\mathrm{nm}$. The uniformity of the interface has been observed by electron microscopy and the mechanical strength has been assessed by adhesion tests. A theoretical model of the processes occurring during laser irradiation has been used to obtain a rationale of the $\mathrm{SiC}$ formation, in terms of interdiffusion of silicon and diamond, after reaching the melting energy thresholds. In the next section we discuss and compare our experimental and theoretical results and in the following ones we discuss the two research lines presently pursued by our collaboration. One line is aimed to develop the prototype of a monolithic SOD radiation detector where the diamond is the sensor connected by Through Silicon Vias (TSV) to the processing electronics. The other line heads to connect the electronic of a MicroElectrode Array (MEA) chip with the living cells of sites placed on a diamond surface via conductive graphite channels through the diamond bulk. This latter implementation opens the way to longer term applications such as muscular and neuronal prostheses.

\section{Laser Enhanced SOD Fabrication}

We bonded $500 \mu \mathrm{mm}$ thick pCVD diamond and 50-300 $\mu \mathrm{m}$ thick (111) Si plates, of surface area $5 \times 5 \mathrm{~mm}^{2}$ by means of a $355 \mathrm{~nm}, 20$ ps radiation from a Nd:YAG laser. Pulses are headed to the Si plate through a fused silica viewport and the diamond sample. The radiation is absorbed in about $20 \mathrm{~nm}$ inside the silicon and melts locally the two materials starting the bonding process, which is based on interdiffusion and final solidification. 
The samples, carefully cleaned, are inserted into a gas-tight chamber under Ar atmosphere in order to avoid oxygen contamination of the silicon-diamond interface. During the irradiation the samples are pressed together with an $80 \mathrm{MPa}$ uniaxial pressure. The laser spot on silicon has a diameter of the order of $1 \mathrm{~mm}$ and an energy density of $1-10 \mathrm{~J} / \mathrm{cm}^{2}$ per pulse. The whole interface is bonded by scanning the laser spot over the whole surface, step by step. At each step we integrate more than one laser pulse (5-50), since a single pulse per step dose not suffice for the bonding.

Surface textures of the samples were measured with white light interferometric microscopy, in order to oppose the smoothest diamond and Si faces. The best diamond surfaces had a roughness height of about $10 \mathrm{~nm}$ and a roughness width of about $100 \mu \mathrm{m}$. The silicon surfaces were an order of magnitude better. Under these conditions, we estimate that the applied pressure ensures the contact of about $80 \%$ of the silicon and diamond surfaces which is good enough for complete bonding.

For all the SOD samples we used state-of-the-art pCVD diamond, but any type of transparent diamond, as the single-crystal one ( $\mathrm{scCVD}$ ), can be used, thus marking a dramatic difference with respect to the more diffused diamond growth onto silicon technique.

Detailed information on the characterization of the obtained SOD material can be found in ref.[16]. Here, we briefly resume the main results and discuss still open problems which require a finer tuning of the process parameters.

Adhesion tests, performed on several SOD samples, have given a mechanical strength higher than $12 \mathrm{MPa}$. Above these limits the tested samples showed either fractures of the silicon bulk, away from the diamond interface, or the detachment from the test gadget. The inspection by Scanning Electron Microscopy (SEM) of the SOD cross sections, at relatively low magnifications, has demonstrated the uniformity of the bonding on a micrometer scale. In more detail, Raman spectroscopy, performed on different samples, has evidenced the presence of an amorphous silicon (a-Si) layer at the interface with a mean thickness of $85 \mathrm{~nm}$. The presence of amorphous carbon in some points of the silicon-diamond interface has also been observed, but it has not been possible to quantify it, although the laser line used were the $\mathrm{Kr} 647.1 \mathrm{~nm}$ and $752.5 \mathrm{~nm}$ lines, which should enhance the $s p^{2}$ diamond bonding. Analyses with the Fourier Transform Infrared (FTIR) spectroscopy have shown the presence of amorphous or nanocrystalline silicon carbide ( $\mathrm{SiC}$ ). The $\mathrm{SiC}$ effective layer width depends on the number of laser shots per position: from $50 \mathrm{~nm}$ to $3 \mathrm{~nm}$, passing from 50 laser pulses to 10, respectively. The results obtained with the optical analyses are confirmed by preliminary results of Scanning Transmission Electron Microscopy (STEM), performed on $20 \mathrm{~nm}$ SOD layers, obtained by thinning with focused ion beam. An interface layer of about $100 \mathrm{~nm}$ has been clearly observed. A chemical characterization performed on this interface region by Energy Dispersion X-ray analysis (EDX) has evidenced a gradual transition from the silicon to the carbon phase.

A recently developed theoretical model of the process occurring during the laser pulse (20 ps) and in the subsequent cooling period (some nanoseconds) gives a deeper insight into the physics involved. The model will be the subject of a fore coming paper, here we limit ourselves to outline its main results.

The model input parameters are the pulse width, the laser wavelength and the energy per pulse. The unidimensional model divides the region in proximity of the interface into layers and, for each layer, takes into account reflection and absorption of the laser beam, diffusion and recombination of 
the electron-hole pairs created by the absorption and energy transfer from the plasma of the excited pairs to the lattice. Temperature, pressure and phase fields are then numerically calculated in the region of interest. The main output values are: the energy threshold of the incoming laser pulse to obtain the melting of both $\mathrm{Si}$ and diamond at the interface and the depth of the interested region.

The performed calculations indicates that both depth and energy threshold increase with the laser pulse width. At the used wavelength $\left(355 \mathrm{~nm}\right.$ ), the energy threshold is $E_{20 \mathrm{ps}}=0.25 \mathrm{~J} / \mathrm{cm}^{2}$ for a 20 ps pulse width and about $E_{7 \mathrm{~ns}}=3.3 \mathrm{~J} / \mathrm{cm}^{2}$ for a $7 \mathrm{~ns}$ pulse. Correspondingly, the expected region depth goes from a $50 \mathrm{~nm}$ minimum for the $20 \mathrm{ps}$ pulse to $400 \mathrm{~nm}$ for the $7 \mathrm{~ns}$ pulse. The model needs to be further validated, nevertheless it is quite consistent with our optical spectroscopy and electron microscopy results obtained so far. It points out that we can about halve the interface depth by decreasing the pulse energy from the present $1-2 \mathrm{~J} / \mathrm{cm}^{2}$ to $E_{20 \mathrm{ps}}=0.25 \mathrm{~J} / \mathrm{cm}^{2}$ but we can decrease it also using faster laser pulses, i.e., in the domain of hundreds of femtosecond. One more indication of the validity of our model is the observation that all the bonding processes operated above the computed melting thresholds of diamond were successful, while the one operated below failed.

\section{SOD Monolithic Pixel Detectors}

The development of high resolution sensors has involved a strong research effort during the last three decades[8]. Pixel sensors are routinely used in optical imaging for commercial purposes, but their application in High Energy Physics (HEP) and space experiments is still a subject of intense investigation. Three are the main issues of the development of the pixel technology. One is the pixel sensitive volume which is correlated to the signal-to-noise ratio $(\mathrm{S} / \mathrm{N})$ performances. One other is the pixel density, which implies read out complexity and power budget, mostly in case of large detector mosaics. The last issue concerns the radiation resistance of the detector both for the sensor and the electronics parts.

In HEP applications, the technology developments have led mainly to hybrid systems with external read out chips connected to the sensor volume by means of bump-bonding processes. Pixel detectors are deployed in the inner part of the HEP detectors (less than $20 \mathrm{~cm}$ from the interaction point) where more useful is their high geometric resolution. Looking forward to the next hadronic colliders (SLHC), to their higher beam luminosities and background radiation, the development of the pixel technology seems mandatory. Of relevance is the choice of the material, with regards to which several $R \& D$ activities are ongoing. Solutions to increase the resistance of silicon are under study, but also the diamond option has arisen an increasing interest. The diamond industrial quality has been constantly growing, while its cost remains high. But large scale applications can lower it in the future and, however, pixel detectors remain confined in small/moderate volumes around collider interaction points.

Another interesting item for the pixel detector development is the growth of the 3D integrated electronics technique, where several thin layers (tiers) are stacked together and connected by Through Silicon Via (TSV). Each layer has its functionality and its technology. 3D stacks sitting on an optical sensor with all silicon material are now in agenda.

We believe indeed that our SOD technique is a good opportunity to build up a 3D stack based on a diamond sensor. At present we intend to limit ourselves to an unique silicon layer, the read out 


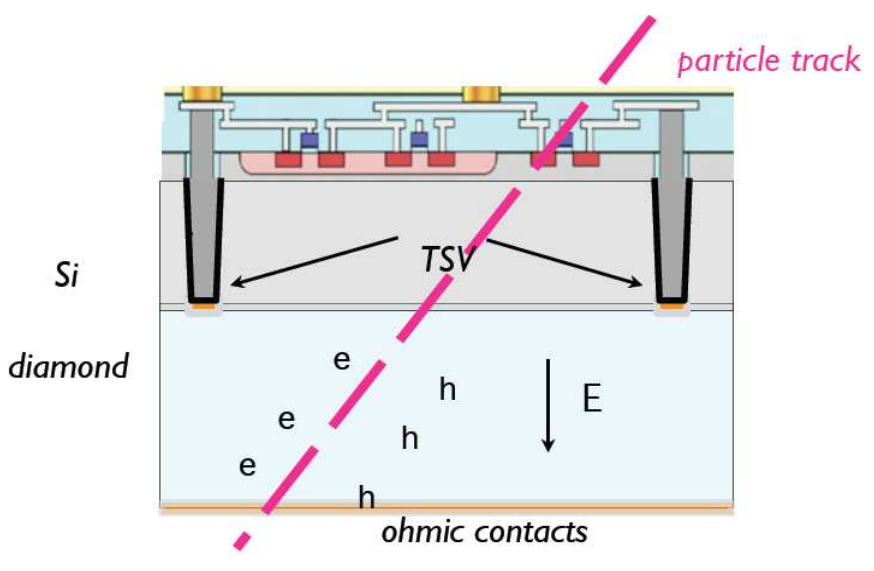

Figure 1: Schematics of a monolithic SOD detector. Ohmic contacts are coated on the two diamond sides ensuring the required polarization. Collection of the radiation generated charge is obtained at the contacts which are linked via the TSV to the front-end electronics on the silicon side of the SOD chip.

electronics chip. The aim of the project is sketched in Fig. 1 and it is addressed to HEP applications, but the technology can be applied in any field where diamond plays the role of sensor.

The fabrication of TSV is the main issue of this line of research. Since we want to bond directly an electronic die to diamond, pre or post-processing implementation of TSV has to be followed by ohmic contact fabrication at the Si-diamond interface. To investigate and to set up the suitable procedures, we foresee an intermediate step: to implement SOD devices equipped only with silicon TSV matrix soldered to the diamond ohmic contacts at the interface (see Fig. 2). The fabrication of ohmic contacts is one of the most challenging aspects of the implementation, the other demanding aspects concerning the TSV realization. By the implementation and refinement of this intermediate step it will be possible to obtain the device depicted in Fig. 1.

\section{SOD Biological Interfaces}

The use of diamond in bioelectrochemical applications can be very promising for the present SOD technique. All the interest stems from the electrochemical properties and the biocompatibility of diamond and from the necessity of integration of the microprobe implants with the signal elaboration electronics. The integration is needed to fulfill the request for small device dimensions and reliable real-time data elaboration. The use of micro electrode arrays (MEAs) for measuring and evoking the extracellular, electro-physiologic activity is now increasing for both in-vivo and in-vitro applications. The electrodes have different shapes in the two cases, needle-like in the former, pad-like in the latter, but, in both cases, they must exhibit as low as possible impedances up to few $\mathrm{kHz}(<1 \mathrm{M} \Omega)$ and a very good stability, to ensure registration and stimulation of neuronal activity for long periods, from several months to several years.

The most typical probe materials are the noble metals and some nano structured materials such TiN and black-Pt. More recently, interest rose in carbon nanotubes (CNT). Nevertheless, diamond is a very interesting material for MEAs, it offers unique interface properties between electrode (diamond) and electrolyte, superior to any other material used nowadays. Semiconducting boron-doped 


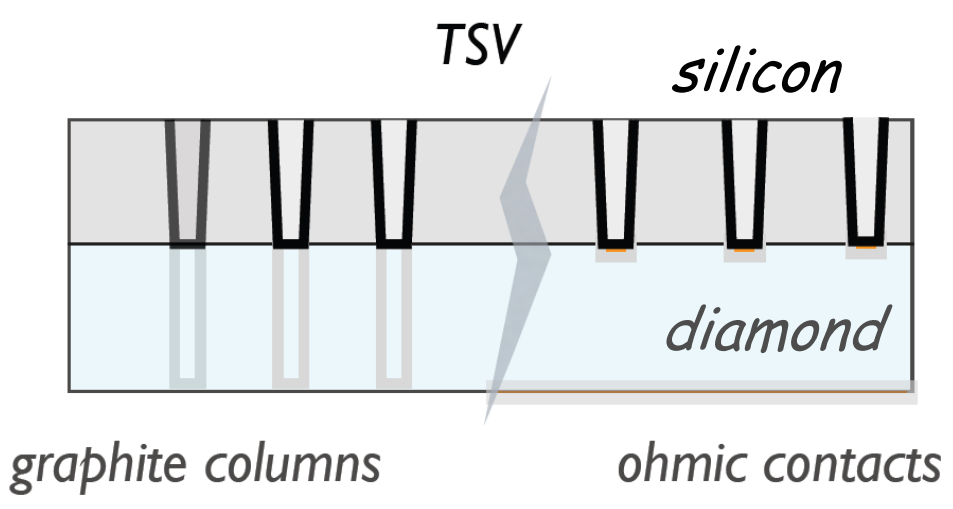

Figure 2: Two basic SOD structures to implement: TSVs for a SOD detector (right) and TSVs with their graphite channel extensions for the bio-SOD (left)

diamond possess a large potential window between the oxygen and hydrogen peaks. This means a wider range of applied potential between diamond and electrolyte, which does not result in redox reactions and allows the application of stimuli to neurons in a more effective and non-toxic way. Other appealing characteristics of diamond are the low background current during electrochemical measurements, due to its adsorption inertia, the low interface capacitance with aqueous solutions and the independence from $\mathrm{pH}$. All these aspects makes diamond a very interesting material to be worked for living cell adhesion.

In this context, our SOD technique can be an ideal approach to implement biosensors with integrated micro-electrodes on the diamond side and the electronics on the silicon side. But a further issue other than the above mentioned TSVs must be fixed. It concerns the ohmic connection between points on the two interfaces the diamond, at the living cell side and at the silicon side, i. e., each micro electrode must be connected via a conductive channel to its TSV and its electronics on the other side of diamond.

Two are the ways of implementing such conductive channels all based on deep through diamond graphite columns. The first starts with the formation of a graphitic seed at some micrometers below the diamond surface as it is obtained by implantation and successive annealing. After that the graphitic seed is extended to form a graphite column by irradiation[17] with a nanosecond 532 $\mathrm{nm}$ laser. Laser pulses are absorbed at the end of the graphite zone producing the diamond melting and its propagation to the opposite surface. Upon cooling highly conducting graphitic channel, about $10 \mu \mathrm{m}$ in radius is formed, whose resistivity is about $10^{-3} \Omega \mathrm{m}$. The second method, recently reported[18], makes only use of a femtosecond laser, focused inside the diamond along its thickness, step by step, so to form a long graphite microchannel $(\approx 1 \mathrm{~mm})$. No electrical characterization of such microchannels has been reported. This is critical for our application, since a low noise via through the diamond should not exceed a resistance of about $100 \Omega$.

The achievement of laser graphitization with different pulse width values can be explained by the fact that it is a threshold process ruled by the net energy density per pulse.

After TSVs the conductive channels in diamond are the base milestone to reach, in order to project a full prototype of a biological SOD device. Fig. 2 showns the two basic SOD devices 


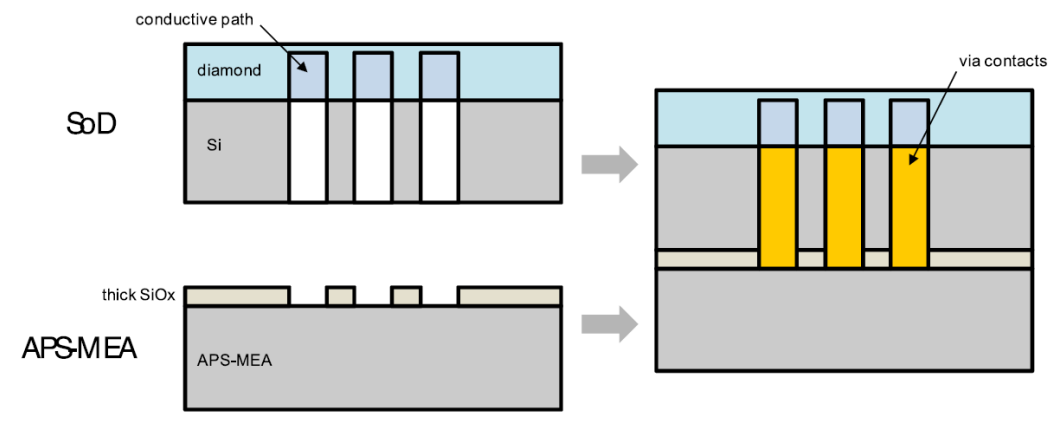

Figure 3: Schematic description of a SOD-MEA. An APS-MEA chip is interconnected with a SOD chip, to exploit the unique bioelectrochemical properties of diamond. The APS-MEA $20 \mu \mathrm{m} \times \mu \mathrm{m}$ electrode size can be used as well for the Si vias of the SOD and the diamond electrodes.

to implement: TSVs for a SOD detector and TSVs with their graphite channel extensions for the bio-SOD device.

Because of the complexity of the devices, we plan to focus our first efforts on the characterization of the diamond biological material interface and on its connection to the opposite silicon interface and its TSVs. In order to understand the functionality of such new SOD device in in-vitro measurements we plan to connect it to existing MEA chips by means of a flip-chip technique. The chip to use is the APS-MEA recently developed [19] and illustrated in Fig. 3. The APS chip, implemented with a CMOS technology, exhibits a matrix of 4096, $20 \times 20 \mu \mathrm{m}^{2}$ pixels, fully equipped with electronics which allows an high spatial resolution, together with a relatively high sampling frequency $(8 \mathrm{kHz})$. The capability of this device to record electrophysiological signals with an unprecedented resolution has been recently demonstrated in-vitro [20].

The SOD-MEA system depicted in Fig. 3 represents an important benchmark in the short term, for future developments, in the long term, of more dedicated 3D-LSI architectures

\section{References}

[1] G. K. Celler, S. Cristoloveanu, Frontiers of silicon-on-insulator, J. Appl. Phys. 93 (2003) 4955.

[2] K. Raleva, D. Vasileska, S. M. Goodnick, Is SOD technology the solution to heating problems in SOI devices?, IEEE Trans. Electron Devices 29 (2008) 621.

[3] J.-P. Mazellier, O. Faynot, S. Cristoloveanu, S. Deleonibus, P. Bergonzo, Integration of diamond in fully-depleted silicon-on-insulator technology as buried insulator: A theoretical analysis, Diamond Relat. Mater. 17 (2008) 1248.

[4] D. Francis, F. Faili, D. Babic, F. Ejeckam, A. Nurmikko, H. Maris, Formation and characterization of 4-inch GaN-on-diamond substrates, Diamond Relat. Mater., 2009, article in press.

[5] A. Aleksov, X. Li, N. Govindaraju, J. Gobien, S. Wolter, J. Prater, Z. Sitar, Silicon-on-diamond: An advanced silicon-on-insulator technology, Diamond Relat. Mater. 14 (2005) 308.

[6] A. Aleksov, J. Gobien, X. Li, J. Prater, Z. Sitar, Silicon-on-diamond an engineered substrate for electronic applications, Diamond Relat. Mater. 15 (2006) 248. 
[7] A. Kaiser, D. Kueck, P. Benkart, A. Munding, G. Prinz, A. Heittmann, H. Huebner, R. Sauer, E. Kohn, Concept for diamond 3-D integrated UV sensor, Diamond Relat. Mater. 15 (2006) 1967.

[8] N. Wermes, Pixel detectors for charged particles, Nucl. Instrum. Methods Phys. Res. A 604 (2009) 370.

[9] A. BenMoussa, A. Theissen, F. Scholze, J. F. Hochedez, U. Schühle, W. Schmutz, et al. Performance of diamond detectors for VUV applications, Nucl. Instrum. Methods Phys. Res. A 568 (2006) 398

[10] M. Rebisz-Pomorska, D. Tromson, A. Isambert, B. Marczewska, and P. Bergonzo, Single crystal chemical vapor deposited diamond detectors for intensity-modulated radiation therapy applications, J. Appl. Phys. 106 (2009) 084509.

[11] P. Ariano, A. L. Giudice, A. Marcantoni, E. Vittone, E. Carbone, D. Lovisolo, A diamond-based biosensor for the recording of neuronal activity, Biosens. Bioelectron. 24 (2009) 2046.

[12] M. Bonnauron, S. Saada, L. Rousseau, G. Lissorgues, C. Mer, P. Bergonzo, High aspect ratio diamond microelectrode array for neuronal activity measurements, Diamond Relat. Mater. 17 (2008) 1399.

[13] G. N. Yushin, S. D. Wolter, A. V. Kvit, R. Collazo, B. R. Stoner, J. T. Prater, Z. Sitar, Study of fusion bonding of diamond to silicon for silicon-on-diamond technology, Appl. Phys. Lett. 81 (2002) 3275.

[14] G. Yushin, A. Aleksov, S. Wolter, F. O. , J. Prater, Z. Sitar, Wafer bonding of highly oriented diamond to silicon, Diamond Relat. Mater. 13 (2004) 1816.

[15] J.-P. Mazellier, J. Widiez, F. Andrieu, M. Lions, S. Saada, M. Hasegawa, K. Tsugawa, L. Brevard, J. Dechamp, M. Rabarot, V. Delaye, S. Cristoloveanu, L. Clavelier, S. Deleonibus, P. Bergonzo, O. Faynot, First demonstration of heat dissipation improvement in CMOS technology using Silicon-On-Diamond (SOD) substrates, in proceedings of SOI Conference, 2009 IEEE International Foster City, CA, USA.

[16] S. Lagomarsino, G. Parrini, S. Sciortino, M. Santoro, M. Citroni, M. Vannoni, A. Fossati, F. Gorelli, G. Molesini, and A. Scorzoni, Silicon-on-diamond material by pulsed laser technique, Appl. Phys. Lett. 96 (2010) 031901.

[17] R. Walker, S. Prawer, D. N. Jamieson and K. W. Nugent and R. Kalish, Formation of buried p-type conducting layers in diamond 71 (1997) 1492-1494.

[18] T. V. Kononenko, M. S. Komlenok, V. P. Pashinin, S. M. Pimenov, V. I. Konov, M. Neff, V. Romano, W. Lüthy, Femtosecond laser microstructuring in the bulk of diamond, Diamond Relat. Mater. 18 (2008) 196-199.

[19] K. Imfeld, S. Neukom, A. Maccione, Y. Bornat, S. Martinoia, P.-A. Farine, M. Koudelka-Hep, L. Berdondini, Large-scale, high-resolution data acquisition system for extracellular recording of electrophysiological activity, IEEE Trans. Biomed. Eng. 55 (2008) 2064.

[20] L. Berdondini, K. Imfeld, A. Maccione, M. Tedesco, S. Neukom, M. Koudelka-Hep, S. Martinoia, Active pixel sensor array for high spatio-temporal resolution electrophysiological recordings from single cell to large scale neuronal networks, Lab On Chip 9 (2009) 2644-2651. 